\title{
Sex-Specific Differences in the Efficacy of Traditional Low Frequency Versus High Frequency Spinal Cord Stimulation for Chronic Pain
}

\author{
Rosalynn RZ Conic ( $\nabla$ rosalynn.conic@gmail.com ) \\ UCSD: University of California San Diego https://orcid.org/0000-0002-9209-2883 \\ Jacob Caylor \\ Northwest Pain Care \\ Christina L. Cui \\ Duke University Department of Surgery \\ Zabrina Reyes \\ University of California San Diego School of Medicine \\ Eric Nelson \\ Pacific Western University of Health Sciences \\ Sopyda Yin \\ University of California San Diego \\ Imanuel Lerman \\ University of California San Diego https://orcid.org/0000-0002-6564-7222
}

\section{Research Article}

Keywords: spinal cord stimulator, outcomes, HF-SCS, LF-SCS, VAS, opioid, sex

Posted Date: February 24th, 2022

DOI: https://doi.org/10.21203/rs.3.rs-1335922/v1

License: (9) This work is licensed under a Creative Commons Attribution 4.0 International License. Read Full License 


\section{Abstract}

\section{Introduction:}

Spinal cord stimulation (SCS), an FDA-approved therapy for chronic pain, uses paresthesia (low frequency SCS (LF-SCS)) or paresthesia-free (high frequency SCS (HF-SCS)) systems, providing analgesia through partially-elucidated mechanisms, with recent studies indicating a sexual dimorphism in pain pathogenesis. We aim to evaluate SCS therapy sex effects based on paradigm, utilizing visual analog scores (VAS), perceived pain reduction (PPR), and opioid use.

\section{Methods:}

A retrospective cohort study of SCS patients implanted between 2004-2020 ( $n=237)$ was conducted. Descriptive statistics and linear mixed methods analyses were used.

\section{Results:}

HF-SCS was implanted in 94 patients ( 40 females, 54 males), and LF-SCS in 143 (70 females, 73 males). At 3 months and 6 months, HF-SCS $(p<0.001)$ and LF-SCS $(p<0.005)$ had lower VAS scores compared to baseline $(p<0.005)$, with no differences across groups. PPR improved in both post-implantation $(p<0.006)$ and at 3 months $(p<0.004$ respectively), compared to baseline persisting to $6(p<0.003)$ and 12 months $(p<0.01)$ for HF-SCS, with significantly better PPR for HF-SCS at 3 $(p<0.008)$ and $6(p<0.001)$ months compared to LF-SCS. LF-SCS patients used more opioids at every time point $(p<0.05)$, with no differences compared to baseline.

VAS for HF-SCS males, HF-SCS and LF-SCS females improved at $3(p=0.001)$ and 6 months $(p<0.05)$, and at 3 months for LF-SCS males $(p<0.001)$. Female HF-SCS had improved PPR at $3(p=0.016)$ and $6(p=0.022)$ months compared to baseline, and at $6(p=0.004)$ months compared to LF-SCS. Male HF-SCS and LF-SCS had improved PPR post-trial $(p<0.05)$ and at 3 months $(p<0.05)$, with HF-SCS having greater benefit at $3(p<0.05)$ and $6(p<0.05)$ months. LF-SCS males but not females used less opioids at 6 months $(p=0.017)$ compared to baseline; however this effect did not persist.

On linear mixed model analyses, including age, sex and stimulator type, VAS decreased with age and at each timepoint, with a trend towards increasing with sex, while PPR increased at 3 and 6 months and opioid use was decreased with HF-SCS use.

\section{Discussion:}

PPR at 3 and 6 months improved to a greater extent in HF-SCS. HF-SCS females had improved PPR at 3 and 6 months, and only LF-SCS males used less opioids at 6 months, potentially indicating sex-based pathway. Future studies should further elucidate differences in sex-based pathways and identify optimal SCS opioid-sparing paradigms for chronic pain patients.

\section{Introduction}

Chronic pain, which is defined as pain persisting for more than six months, affects one in five Americans and can lead to reduced mobility and function as well as depression anxiety and other psychosocial changes [1, 2]. The standard treatment is medical management with a combination of non-steroid anti-inflammatory drugs, muscle relaxants and opioids; however, given the high risk for adverse outcomes with long-term opioid therapy, other alternatives are needed [3, 4].

One promising potential therapeutic for chronic pain; spinal cord stimulation (SCS), is indicated for chronic pain conditions including Failed Back Surgery Syndrome (FBSS), Complex Regional Pain Syndrome (CRPS), radicular and nerve root pain, postherpetic neuralgia, pain due to peripheral nerve injury, and intercostal neuralgia and phantom limb pain [5]. SCS systems are implanted in the epidural space and deliver electrical pulses to decrease or block transmission of pain signals at spinal segmental and supraspinal levels [6]. In traditional SCS, also known as low-frequency SCS (LF-SCS) or paresthesia-based SCS, the electrical pulses range between $2-60 \mathrm{~Hz}$, most often in the $40-60 \mathrm{~Hz}$ range [6-8]. But LF-SCS can be limited by 
anatomical restrictions that reduce or abolish coverage to certain targets, such as lower back and foot, while patient tolerance of paresthesia and loss of efficacy over time is common [9]. Paresthesia free SCS (PF-SCS), a relatively recent FDA approved SCS paradigm known as high-frequency SCS (HF-SCS), that delivers $10,000 \mathrm{~Hz}$ pulses consistently improves pain relief (for up to 36 months) [10] and to a greater extent when compared to LF-SCS (for up to 2 years) [6, 11]. Both LF-SCS and HF-SCS implantation is associated with decrease in opioid use [6,12], reduced disability [13-15], while others have shown and improved sleep $[15,16]$.

Given the recent discovery of sex-specific endogenous pain pathways [17], there is considerable interest in sex-based SCS efficacy. Preclinical models show that male allodynia can develop via a testosterone-dependent glial cell pathway [18] while females utilize a T-cell-dependent mechanism (Figure 1)[19]. Clinically, females respond differently to analgesics while they are more likely to have an SCS explanted due to inadequate pain relief compared to males; however, sex specific SCS response remains understudied [20-23]. With few reports that identify interactions between sex and SCS we aimed to fill this knowledge gap through a single site (University of California San Diego), large ( $n=237$ ) retrospective (2004-2020) analyses that compared SCS paradigm (LF-SCS vs HF-SCS), efficacy (pain relief and opiate sparing effects) across sex[24].

\section{Methods}

This study was approved by the institutional review board at the University of California San Diego (IRB\#20031). A retrospective chart review of patients who underwent LF-SCS and HF-SCS trials and implantation at the University of California San Diego between October 2004 and February 2020 was performed $(n=237)$. Patients who did not undergo permanent implantation, patients without demographic data and those with multiple pain conditions requiring separate treatment were excluded. Data regarding patient age, sex, primary diagnosis, type of SCS implant, revision, and explant were collected. Outcome data were collected 3 months prior to trial (baseline), within 1-2 weeks after implantation (postimplantation) and at 3, 6, and 12 months consisted of visual analogue scores (VAS) for pain (rated from 0 - 10), perceived pain reduction attributable to the SCS (PPR, rated from 0 - 100 as a percentage), and opioid dosage converted into morphine equivalents (continuous)[25].

Chi square test and Fisher exact test were used to analyze categorical data. Paired t-test was used to compare the raw pain change between each time point and the baseline measurement. T-test was used to evaluate raw pain change between stimulator types across each time point. Linear mixed models were used to account for repeated measures for each outcome. Results were stratified by type of stimulation paradigm (LF-SCS vs HF-SCS) and sex. R statistical software version 4.1.1 was used for data analysis [26]. The dataset analyzed in this study is available from the corresponding author on reasonable request.

\section{Results}

There were 237 patients total, of whom 143 patients were implanted with an LF-SCS and 94 who were implanted with an HFSCS (Table 1). Patients implanted with HF-SCS tended to be older $(61.02 \pm 14.48$ vs $54.07 \pm 15.05, \mathrm{p}<0.001)$ and Non-Hispanic (84\% vs $77.6 \%, p=0.03)$. The most common primary indication for implant was FBSS in both groups (44.8\% LF-SCS vs. $39.4 \%$ HF-SCS), followed by lumbar radiculopathy (21\% LF-SCS vs. $21.3 \%$ HF-SCS), CRPS (16.1\% LF-SCS vs. $7.4 \%$ HF-SCS) and lumbar spondylosis (1.4\% LF-SCS vs $14.9 \%$ HF-SCS). 
Table 1

Patient Characteristics by Type of Stimulator

\begin{tabular}{|c|c|c|c|}
\hline & HF-SCS & LF-SCS & p-value \\
\hline $\mathrm{n}$ & 94 & 143 & \\
\hline Sex $=$ Male $(\%)$ & $54(57.4)$ & $70(49.0)$ & 0.251 \\
\hline Age (mean (SD)) & $61.02(14.48)$ & $54.07(15.05)$ & $<0.001$ \\
\hline Ethnicity (\%) & & & 0.027 \\
\hline African American & $0(0.0)$ & $2(1.4)$ & \\
\hline Caucasian & $3(3.2)$ & $17(11.9)$ & \\
\hline Hispanic & $9(9.6)$ & $5(3.5)$ & \\
\hline Non-Hispanic & $79(84.0)$ & $111(77.6)$ & \\
\hline Unknown (Patient cannot or refuses to declare ethnicity) & $3(3.2)$ & $8(5.6)$ & \\
\hline Marriage Status (\%) & & & 0.838 \\
\hline Married & $60(63.8)$ & $89(62.2)$ & \\
\hline Other/Unknown & $1(1.1)$ & $2(1.4)$ & \\
\hline Separated/Divorced & $13(13.8)$ & $19(13.3)$ & \\
\hline Single & $16(17.0)$ & $30(21.0)$ & \\
\hline Widowed & $4(4.3)$ & $3(2.1)$ & \\
\hline Payor Name (\%) & & & $<0.001$ \\
\hline Medicare & 30 ( 31.9$)$ & $23(16.1)$ & \\
\hline None/Unknown & $49(52.1)$ & $84(58.7)$ & \\
\hline Other Government & $8(8.5)$ & $3(2.1)$ & \\
\hline Private Insurance & $7(7.4)$ & $28(19.6)$ & \\
\hline Workman's Compensation & $0(0.0)$ & $5(3.5)$ & \\
\hline Primary Diagnosis for Implant (\%) & & & $<0.001$ \\
\hline Complex Regional Pain Syndrome & $7(7.4)$ & $23(16.1)$ & \\
\hline Cranial Neuropathy & $2(2.1)$ & $5(3.5)$ & \\
\hline Failed Back Surgery Syndrome & $37(39.4)$ & $64(44.8)$ & \\
\hline Lumbar Radiculopathy & $20(21.3)$ & $30(21.0)$ & \\
\hline Lumbar Spondylosis & $14(14.9)$ & $2(1.4)$ & \\
\hline Neuropathic Pain & $7(7.4)$ & 17 ( 11.9) & \\
\hline Other Chronic Pain & $7(7.4)$ & $2(1.4)$ & \\
\hline Revision or Explant (\%) & $25(17.5)$ & $15(16.0)$ & 0.897 \\
\hline Patient status (\%) & & & 0.016 \\
\hline Alive & $78(83.0)$ & $120(83.9)$ & \\
\hline
\end{tabular}




\begin{tabular}{|llll|}
\hline & HF-SCS & LF-SCS & P-value \\
\hline Deceased & $0(0.0)$ & $9(6.3)$ \\
\hline Unknown & $16(17.0)$ & $14(9.8)$ & \\
\hline
\end{tabular}

HF-SCS and LF-SCS patients had significantly lower VAS compared to baseline at 3 months (HF-SCS 4.25 \pm 2.3 , p<0.001; LFSCS 4.87 $\pm 2.48, p<0.001$ ) and 6 months (HF-SCS 4.91 $\pm 2.42, p<0.001$; LF-SCS 5.71 $\pm 2.17, p=0.005$ ) after implantation; however this effect did not survive to 12 months and there were no differences across the two groups $(3$ months $p=0.18,6$ months $p=0.13$, Figure 2a, Table 2). Both HF-SCS and LF-SCS had better PPR post-implantation (HF-SCS 55.41 \pm 27.54 ), $p=0.006$; LF-SCS 48.17 $\pm 29.84, p<0.001$ ) and at 3 months (HF-SCS 61.18 $\pm 25.35, p<0.001$; LF-SCS 47.93 $\pm 28.12, p=0.004$ ); however this effect was sustained to 6 (HF-SCS 58.38 \pm 26.54 , $p=0.003$; LF-SCS 33.70 $\pm 25.94, p=0.09$ ) and 12 months (HFSCS 57.17 $\pm 28.44, p<0.001$; LF-SCS $42.50 \pm 26.75, p=0.052$ ) only for the HF-SCS group. In addition, HF-SCS patients had significantly improved PPR compared to LF-SCS patients at 3 and 6 months ( $p=0.008, p<0.001$ respectively, Figure $2 b$ ). There were no differences in opioid use in HF-SCS or LF-SCS patients compared to baseline; however patients with LF-SCS required significantly more opioids compared to HF-SCS at every time point (baseline 105.44 \pm 118.49 LF-SCS vs. 41.45 \pm 60.27 HF-SCS, $p<0.001$; post-implantation 107.91 \pm 142.99 LF-SCS vs. $35.15 \pm 51.72$ HF-SCS, $p<0.001 ; 3$ months $110.23 \pm 197.08$ LF-SCS vs $47.63 \pm 97.28$ HF-SCS, $p=0.027 ; 6$ months $112.98 \pm 142.97$ LF-SCS vs. $19.67 \pm 24.96$ HF-SCS, $p<0.001$ and 12 months $106.03 \pm 146.08$ LF-SCS vs. $33.92 \pm 48.66$ HF-SCS, $p=0.014)$. Nine patients died during this study, all of whom were in the LF-SCS group. There were no differences in sex (49\% LF-SCS vs. $57.4 \%$ HF-SCS, $p=0.25$ ), marriage status ( $p=0.84)$, and revisions or explants ( $16 \%$ LF-SCS vs. $17.5 \%$ HF-SCS, $p=0.89$ ) between the two groups. 
Table 2

Visual analog scale scores, perceived pain reduction and opioid use by type of stimulator. P-values are based on paired t-test between baseline and each individual time point based on stimulator type. *p-value compares HF-SCS to LF-SCS at a given time point

\begin{tabular}{|c|c|c|c|c|c|}
\hline & HF-SCS & p-value & LF-SCS & p-value & *p-value across \\
\hline \multicolumn{6}{|l|}{ VAS Scores (mean (SD)) } \\
\hline Baseline & $6.61(1.85)$ & & $6.31(2.09)$ & & 0.26 \\
\hline Post-Implantation & $6.01(2.22)$ & 0.09 & $5.73(2.34)$ & 0.061 & 0.47 \\
\hline 3 Months Post Implant & $4.25(2.3)$ & $<0.001$ & $4.87(2.48)$ & $<0.001$ & 0.18 \\
\hline 6 Months Post Implant & $4.91(2.42)$ & $<0.001$ & $5.71(2.17)$ & 0.005 & 0.127 \\
\hline 12 Months Post Implant & $5.52(2.39)$ & 0.16 & $5.69(1.87)$ & 0.15 & 0.81 \\
\hline \multicolumn{6}{|c|}{ Perceived Pain Reduction (mean (SD)) } \\
\hline Baseline & $31.74(26.31)$ & & $31.21(29.91)$ & & 0.99 \\
\hline Post-Implantation & $55.41(27.54)$ & 0.006 & $48.17(29.84)$ & $<0.001$ & 0.09 \\
\hline 3 Months Post Implant & $61.18(25.35)$ & $<0.001$ & $47.93(28.12)$ & 0.004 & 0.008 \\
\hline 6 Months Post Implant & $58.38(26.54)$ & 0.003 & $33.70(25.94)$ & 0.09 & $<0.001$ \\
\hline 12 Months Post Implant & $57.17(28.44)$ & 0.01 & $42.50(26.75)$ & 0.052 & 0.067 \\
\hline \multicolumn{6}{|c|}{ Morphine mili-Equivalents (mean (SD)) } \\
\hline Baseline & $41.45(60.27)$ & & $105.44(118.49)$ & & $<0.001$ \\
\hline Post-Implantation & $35.15(51.72)$ & 0.99 & $107.91(142.99)$ & 0.8 & $<0.001$ \\
\hline 3 Months Post Implant & $47.63(97.28)$ & 0.21 & 110.23 (197.08) & 0.35 & 0.027 \\
\hline 6 Months Post Implant & $19.67(24.96)$ & 0.12 & 112.98 (142.97) & 0.79 & $<0.001$ \\
\hline 12 Months Post Implant & $33.92(48.66)$ & 0.15 & $106.03(146.08)$ & 0.36 & 0.014 \\
\hline
\end{tabular}

\section{Sex Subset Analysis}

There were 73 females implanted with LF-SCS and 40 with HF-SCS (Table 3). The females implanted with HF-SCS tended to be older (53.70 \pm 14.98 LF-SCS vs $65.03 \pm 12.60$ HF-SCS, $p<0.001)$. LF-SCS females had improved VAS scores post-trial (5.78 $\pm 2.44, p=0.04)$ compared to baseline, and both HF-SCS and LF-SCS patients had improved VAS at 3 (HF-SCS 4.38 $\pm 2.32, p<0.001$; LF-SCS 5.16 $\pm 2.56, p=0.001$ ) and 6 (HF-SCS 5.35 $\pm 2.03, p=0.023$; LF-SCS 6.03 $\pm 1.92, p=0.032$ ) months, which did not survive to 12 months (HF-SCS $5.89 \pm 2.29, p=0.37$; LF-SCS $5.82 \pm 1.91, p=0.35$ ), and there were no differences in VAS between HF-SCS and LF-SCS females (Figure 3a). 
Patient Characteristics by Type of Stimulator and Sex

\begin{tabular}{|c|c|c|c|c|c|c|}
\hline & Female & & & Male & & \\
\hline & HF-SCS & LF-SCS & p-val & HF-SCS & LF-SCS & p-val \\
\hline $\mathrm{n}$ & 40 & 73 & & 54 & 70 & \\
\hline Age (mean (SD)) & $\begin{array}{l}65.03 \\
(12.60)\end{array}$ & $\begin{array}{l}53.70 \\
(14.98)\end{array}$ & $<0.001$ & $\begin{array}{l}58.06 \\
(15.17)\end{array}$ & $\begin{array}{l}54.46 \\
(15.21)\end{array}$ & 0.193 \\
\hline Ethnicity (\%) & & & 0.62 & & & 0.031 \\
\hline African American & $0(0.0)$ & $1(1.4)$ & & $0(0.0)$ & $1(1.4)$ & \\
\hline Caucasian & $2(5.0)$ & $5(6.8)$ & & $1(1.9)$ & $12(17.1)$ & \\
\hline Hispanic & $1(2.5)$ & $1(1.4)$ & & $8(14.8)$ & $4(5.7)$ & \\
\hline Non-Hispanic & 37 (92.5) & $63(86.3)$ & & $42(77.8)$ & 48 ( 68.6) & \\
\hline $\begin{array}{l}\text { Unknown (Patient cannot or refuses to } \\
\text { declare ethnicity) }\end{array}$ & $0(0.0)$ & $3(4.1)$ & & $3(5.6)$ & $5(7.1)$ & \\
\hline Marriage Status (\%) & & & 0.73 & & & 0.79 \\
\hline Married & $24(60.0)$ & $42(57.5)$ & & $36(66.7)$ & $47(67.1)$ & \\
\hline Other/Unknown & $0(0.0)$ & $0(0.0)$ & & $1(1.9)$ & $2(2.9)$ & \\
\hline Separated/Divorced & $8(20.0)$ & $14(19.2)$ & & $5(9.3)$ & $5(7.1)$ & \\
\hline Single & $5(12.5)$ & $14(19.2)$ & & $11(20.4)$ & $16(22.9)$ & \\
\hline Widowed & $3(7.5)$ & $3(4.1)$ & & $1(1.9)$ & $0(0.0)$ & \\
\hline Insurance Type (\%) & & & 0.07 & & & 0.018 \\
\hline Medicare & $15(37.5)$ & $12(16.4)$ & & $15(27.8)$ & $11(15.7)$ & \\
\hline None/Unknown & $19(47.5)$ & 39 ( 53.4) & & $30(55.6)$ & 45 ( 64.3) & \\
\hline Other Government & $1(2.5)$ & $1(1.4)$ & & $7(13.0)$ & $2(2.9)$ & \\
\hline Private Insurance & $5(12.5)$ & 18 ( 24.7) & & $2(3.7)$ & $10(14.3)$ & \\
\hline Workman's Compensation & $0(0.0)$ & $3(4.1)$ & & $0(0.0)$ & $2(2.9)$ & \\
\hline Primary Diagnosis for Implant (\%) & & & 0.25 & & & 0.006 \\
\hline Complex Regional Pain Syndrome & $4(10.0)$ & $13(17.8)$ & & $3(5.6)$ & $10(14.3)$ & \\
\hline Cranial Neuropathy & $1(2.5)$ & $2(2.7)$ & & $1(1.9)$ & $3(4.3)$ & \\
\hline Failed Back Surgery Syndrome & $18(45.0)$ & $32(43.8)$ & & $19(35.2)$ & $32(45.7)$ & \\
\hline Lumbar Radiculopathy & $8(20.0)$ & $15(20.5)$ & & $12(22.2)$ & $15(21.4)$ & \\
\hline Lumbar Spondylosis & $5(12.5)$ & $1(1.4)$ & & $9(16.7)$ & $1(1.4)$ & \\
\hline Neuropathic Pain & $3(7.5)$ & $9(12.3)$ & & $4(7.4)$ & $8(11.4)$ & \\
\hline Other Chronic Pain & $1(2.5)$ & $1(1.4)$ & & $6(11.1)$ & $1(1.4)$ & \\
\hline Revision or Explant (\%) & $6(15.0)$ & $17(23.3)$ & 0.42 & $9(16.7)$ & $8(11.4)$ & 0.564 \\
\hline Patient Status (\%) & & & 0.036 & & & 0.198 \\
\hline
\end{tabular}




\begin{tabular}{|lllll|}
\hline & Female & \multicolumn{3}{c|}{ Male } \\
\hline Alive & $33(82.5)$ & $64(87.7)$ & $45(83.3)$ & $56(80.0)$ \\
\hline Deceased & $0(0.0)$ & $5(6.8)$ & $0(0.0)$ & $4(5.7)$ \\
\hline Unknown & $7(17.5)$ & $4(5.5)$ & $9(16.7)$ & $10(14.3)$ \\
\hline
\end{tabular}

Females with HF-SCS had greater PPR compared to baseline at 3 months (HF-SCS 60.71 $\pm 24.91, p=0.016$; LF-SCS 48.45 $\pm 26.63, p=0.053$ ), and 6 months (HF-SCS 63.19 $\pm 28.02, p=0.022$; LF-SCS 33.54 $\pm 28.11, p=0.11$ ), without improvement at 12 months (HF-SCS 59.64 $\pm 28.79, p=0.053$; LF-SCS 44.72 $\pm 29.28, p=0.18$; Table 4, Figure 3c). In addition, HF-SCS females had significantly better PPR scores at 6 months compared to LF-SCS females $(p=0.004)$. While there were no differences in HF-SCS and LF-SCS females comparing each time point to baseline, LF-SCS females required significantly more opioids compared to HF-SCS at baseline (HF-SCS 28.52 \pm 29.13 ; LF-SCS 95.99 \pm 93.76 , p=0.003), post-trial (HF-SCS 25.77 \pm 25.3 ; LFSCS 103.84 $\pm 147.40, p=0.001$ ), 3 months (HF-SCS 28.93 \pm 34.52 ; LF-SCS $89.06 \pm 99.04, p=0.006$ ), and 6 months (HF-SCS 22.05 \pm 30.7 ; LF-SCS 129.1 \pm 166.53 , p=0.011), with a trend for higher opioid use for LF-SCS patients at 12 months (HF-SCS 25.69 \pm 28.55 ; LF-SCS 118.63 $\pm 140.99, p=0.064$; Figure $3 e)$. There were no differences in ethnicity $(p=0.62)$, marriage status $(p=0.73)$, insurance type $(p=0.07)$, primary implant diagnosis $(p=0.25)$ or revision/explant $(p=0.42)$ between the female HFSCS and LF-SCS patients. 
Table 4

Visual analog scale scores, perceived pain reduction and opioid use by type of stimulator. P-values are based on paired t-test between baseline and each individual time point based on stimulator type. *p-value compares HF-SCS to LF-SCS for the specific sex at a given time point

\begin{tabular}{|c|c|c|c|c|c|c|c|c|c|c|}
\hline & Female & & & & & Male & & & & \\
\hline & $\begin{array}{l}\text { HF- } \\
\text { SCS }\end{array}$ & p-val & LF-SCS & p-val & $\begin{array}{l}\mathrm{p}- \\
\mathrm{val}^{*}\end{array}$ & HF-SCS & p-val & LF-SCS & p-val & $\begin{array}{l}\text { p- } \\
\text { val* }^{*}\end{array}$ \\
\hline \multicolumn{11}{|l|}{$\begin{array}{l}\text { VAS Scores } \\
\text { (mean } \\
(\mathrm{SD}))\end{array}$} \\
\hline Baseline & $\begin{array}{l}6.77 \\
(2.03)\end{array}$ & & $6.62(2.13)$ & & 0.67 & $\begin{array}{l}6.49 \\
(1.73)\end{array}$ & & $\begin{array}{l}5.93 \\
(2.01)\end{array}$ & & 0.13 \\
\hline Post-Trial & $\begin{array}{l}6.00 \\
(2.46)\end{array}$ & 0.13 & $5.78(2.44)$ & 0.04 & 0.68 & $\begin{array}{l}6.01 \\
(2.05)\end{array}$ & 0.139 & $\begin{array}{l}5.68 \\
(2.24)\end{array}$ & 0.74 & 0.52 \\
\hline $\begin{array}{l}3 \text { Months } \\
\text { Post } \\
\text { Implant }\end{array}$ & $\begin{array}{l}4.38 \\
(2.32)\end{array}$ & $<0.001$ & $5.16(2.56)$ & 0.001 & 0.26 & $\begin{array}{l}4.14 \\
(2.32)\end{array}$ & $<0.001$ & $\begin{array}{l}4.46 \\
(2.34)\end{array}$ & $<0.001$ & 0.66 \\
\hline $\begin{array}{l}6 \text { Months } \\
\text { Post } \\
\text { Implant }\end{array}$ & $\begin{array}{l}5.35 \\
(2.03)\end{array}$ & 0.023 & 6.03 (1.92) & 0.032 & 0.32 & $\begin{array}{l}4.38 \\
(2.78)\end{array}$ & 0.018 & $\begin{array}{l}5.32 \\
(2.42)\end{array}$ & 0.074 & 0.25 \\
\hline $\begin{array}{l}12 \text { Months } \\
\text { Post } \\
\text { Implant }\end{array}$ & $\begin{array}{l}5.89 \\
(2.29)\end{array}$ & 0.37 & $5.82(1.91)$ & 0.35 & 0.74 & $\begin{array}{l}5.35 \\
(2.6)\end{array}$ & 0.22 & $\begin{array}{l}5.50 \\
(1.83)\end{array}$ & 0.27 & 0.99 \\
\hline \multicolumn{11}{|l|}{$\begin{array}{l}\text { Perceived } \\
\text { Pain } \\
\text { Reduction }\end{array}$} \\
\hline Baseline & $\begin{array}{l}29.82 \\
(30.23)\end{array}$ & & 28.65(30.54) & & 0.96 & $\begin{array}{l}34 \\
(23.32)\end{array}$ & & $\begin{array}{l}34.09 \\
(29.38)\end{array}$ & & 0.96 \\
\hline Post-Trial & $\begin{array}{l}57.03 \\
(32.26)\end{array}$ & 0.053 & $\begin{array}{l}45.11 \\
(31.54)\end{array}$ & 0.014 & 0.12 & $\begin{array}{l}54.19 \\
(23.50)\end{array}$ & 0.049 & $\begin{array}{l}51.10 \\
(28.13)\end{array}$ & 0.023 & 0.45 \\
\hline $\begin{array}{l}3 \text { Months } \\
\text { Post } \\
\text { Implant }\end{array}$ & $\begin{array}{l}60.71 \\
(24.91)\end{array}$ & 0.016 & $\begin{array}{l}48.45 \\
(26.63)\end{array}$ & 0.053 & 0.12 & $\begin{array}{l}61.47 \\
(25.98)\end{array}$ & 0.009 & $\begin{array}{l}47.29 \\
(30.26)\end{array}$ & 0.033 & 0.034 \\
\hline $\begin{array}{l}6 \text { Months } \\
\text { Post } \\
\text { Implant }\end{array}$ & $\begin{array}{l}63.19 \\
(28.02)\end{array}$ & 0.022 & $3.54(28.11)$ & 0.11 & 0.004 & $\begin{array}{l}53.46 \\
(24.61)\end{array}$ & 0.09 & $\begin{array}{l}33.86 \\
(24.00)\end{array}$ & 0.60 & 0.027 \\
\hline $\begin{array}{l}12 \text { Months } \\
\text { Post } \\
\text { Implant }\end{array}$ & $\begin{array}{l}59.64 \\
(28.79)\end{array}$ & 0.053 & $\begin{array}{l}44.72 \\
(29.28)\end{array}$ & 0.18 & 0.22 & $\begin{array}{l}53.33 \\
(29.16)\end{array}$ & 0.19 & $\begin{array}{l}40.00 \\
(24.29)\end{array}$ & 0.18 & 0.23 \\
\hline \multicolumn{11}{|l|}{$\begin{array}{l}\text { Morphine } \\
\text { mili- } \\
\text { Equivalents }\end{array}$} \\
\hline Baseline & $\begin{array}{l}28.52 \\
(29.13)\end{array}$ & & $\begin{array}{l}95.99 \\
(93.76)\end{array}$ & & 0.003 & $\begin{array}{l}49.89 \\
(73.36)\end{array}$ & & $\begin{array}{l}115.77 \\
(140.92)\end{array}$ & & 0.053 \\
\hline Post-Trial & $\begin{array}{l}25.77 \\
(25.3)\end{array}$ & 0.78 & $\begin{array}{l}103.84 \\
(147.40)\end{array}$ & 0.45 & 0.001 & $\begin{array}{l}42.59 \\
(65.11)\end{array}$ & 0.25 & $\begin{array}{l}112.16 \\
(139.25)\end{array}$ & 0.86 & 0.006 \\
\hline $\begin{array}{l}3 \text { Months } \\
\text { Post } \\
\text { Implant }\end{array}$ & $\begin{array}{l}28.93 \\
(34.52)\end{array}$ & 0.11 & $\begin{array}{l}89.06 \\
(99.04)\end{array}$ & 0.48 & 0.006 & $\begin{array}{l}58.54 \\
(119.22)\end{array}$ & 0.37 & $\begin{array}{l}134.37 \\
(267.58)\end{array}$ & 0.87 & 0.19 \\
\hline
\end{tabular}




\begin{tabular}{|c|c|c|c|c|c|c|c|c|c|c|}
\hline & Female & & & & & Male & & & & \\
\hline $\begin{array}{l}6 \text { Months } \\
\text { Post } \\
\text { Implant }\end{array}$ & $\begin{array}{l}22.05 \\
(30.7)\end{array}$ & 0.45 & $\begin{array}{l}129.1 \\
(166.53)\end{array}$ & 0.12 & 0.011 & $\begin{array}{l}17.50 \\
(19.45)\end{array}$ & 0.21 & $\begin{array}{l}96.52 \\
(113.5)\end{array}$ & 0.017 & 0.01 \\
\hline $\begin{array}{l}12 \text { Months } \\
\text { Post } \\
\text { Implant }\end{array}$ & $\begin{array}{l}25.69 \\
(28.55)\end{array}$ & 0.83 & $\begin{array}{l}118.63 \\
(140.99)\end{array}$ & 0.071 & 0.064 & $\begin{array}{l}39.62 \\
(59.27)\end{array}$ & 0.23 & $\begin{array}{l}92.82 \\
(151.8)\end{array}$ & 0.10 & 0.13 \\
\hline
\end{tabular}

Seventy males were implanted with LF-SCS and 54 with HF-SCS. There were more Hispanic patients in the HF-SCS group ( $14.8 \%$ vs $5.7 \%$, p $=0.03$ ). LF-SCS patients had more private insurance compared to HF-SCS (14.3\% vs $3.7 \%)$, while HF-SCS had more Medicare ( $27.8 \%$ vs. $15.7 \%$ ) or other governmental insurance (13\% vs. $2.9 \%$, $p=0.018)$. LF-SCS patients had more FBSS (45.7\% vs $35.2 \%)$ and CRPS (14.3\% vs $5.6 \%)$, while lumbar spondylosis was more common in HF-SCS (16.7\% vs $1.4 \%$, $\mathrm{p}=0.006)$.

HF-SCS and LF-SCS males had improved VAS scores at 3 months (HF-SCS 4.14 $\pm 2.32, p<0.001$; LF-SCS 4.46 $\pm 2.34, p<0.001$ ) compared to baseline, which persisted to 6 months only for HF-SCS (HF-SCS 4.38 $\pm 2.78, p=0.018$; LF-SCS 5.32 \pm 2.42 , $p=0.074$ ), and did not survive to 12 months (HF-SCS 5.35 $\pm 2.6, p=0.022$; LF-SCS $5.50 \pm 1.83, p=0.27$ ), with no differences across groups (Figure 3b). PPR was improved among HF-SCS and LF-SCS males post-trial (HF-SCS 54.19 $\pm 23.50, p=0.049$; LF-SCS 51.10 $\pm 28.13, p=0.023$ ) and at 3 months (HF-SCS 61.47 $\pm 25.98, p=0.009$; LF-SCS 47.29 $\pm 30.26, p=0.033$ ) compared to baseline (Figure $3 d)$. Furthermore, HF-SCS males had significantly better PPR at $3(p=0.034)$ and 6 months (HF-SCS 53.46 $\pm 24.61, p=0.09$; LF-SCS 33.86 $\pm 24.00, p=0.60$ ) compared to LF-SCS males which did not survive to 12 months (HF-SCS $53.33 \pm 29.16, p=0.19 ;$ LF-SCS $40.00 \pm 24.29, p=0.18 ; p=0.23$ across).

LF-SCS males had significant reduction it opioid use at 6 months (LF-SCS $96.52 \pm 113.5, p=0.017$ ) compared to baseline, but this was not present at other time points, or among HF-SCS (6 months HF-SCS $17.50 \pm 19.45 \mathrm{p}=0.21)$ patients. In addition, LF-SCS males required significantly more opioids compared to HF-SCS post-trial (HF-SCS 42.59 \pm 65.11 ; LF-SCS 112.16 $\pm 139.25, p=0.006)$, and at 6 months $(p=0.01)$ but not at 3 (HF-SCS 58.54 \pm 119.22 ; LF-SCS 134.37 $\pm 267.58, p=0.19)$ and 12 months (HF-SCS 39.62 \pm 59.27 ; LF-SCS 92.82 $\pm 151.8, p=0.13$, Figure 3e). There were no differences in age $(p=0.19)$, marriage status (0.79), revision/explant $(p=0.56)$ between the male HF-SCS and LF-SCS patients.

\section{Linear Mixed Model}

In the linear mixed model including age, sex, stimulator type, and time, VAS decreased with age $(-0.015,95 \% \mathrm{Cl}-0.0003-(-$ $0.03), \mathrm{p}=0.047)$, at each time point, with the strongest effects seen at 3 months, (post implantation $-0.14,95 \% \mathrm{Cl}-0.21-(-1.16)$, 3 months $-1.1,95 \% \mathrm{Cl}-1.09-(-2.12) ; 6$ months $-1.0,95 \% \mathrm{Cl}-0.33-(-1.41), 12$ months $-0.26,95 \% \mathrm{Cl}-0.24-(-1.43), \mathrm{p}<0.001)$, and a trend towards increasing for female sex $(0.44,95 \% \mathrm{Cl}-0.003-0.88, \mathrm{p}=0.053)$, with a trend towards significant interaction between stimulator type and time $(p=0.068)$.

In the same model for PPR, 3 months $(4.23,95 \% \mathrm{Cl} 0.71-7.78)$ and 6 months $(6.17,95 \% \mathrm{Cl} 2.37-9.99)$ were associated with higher PPR $(p<0.001)$, while HF-SCS was associated with lower PPR $(-4.95,95 \% \mathrm{Cl}-8.36-(-1.5), p=0.005)$, and there was a significant interaction between stimulator type and time $(p=0.027)$.

Finally, in the same model for opioid use, HF-SCS was associated with lower opioid use $(-70.02,95 \% \mathrm{Cl}-23.75-(-116.294)$, $p=0<0.001$ ), without significant effect of age, sex, time or the interaction between time and stimulator type.

\section{Discussion}

This single center retrospective study found significant improvement in VAS at 3 and 6 months which is no longer present at 12 months. LF-SCS and HF-SCS patients had improved PPR post-implantation and at 3 months, which persisted to 6 and 12 months for HF-SCS patients only. Furthermore, patients with HF-SCS have better pain control according to PPR at 3 and 6 
months compared to the LF-SCS group. Some of these differences might be attributed to sex as only HF-SCS females had improved PPR at 3 and 6 months compared to baseline, while both HF-SCS and LF-SCS males had improvement post-trial and at 3 months. Furthermore HF-SCS males had significantly better PPR at 3 and 6 months compared to LF-SCS males while this was only present at 6 months for females. Overall LF-SCS patients used significantly more opioids at each time point compared to those with HF-SCS; however LF-SCS males used less opioids at 6 months, further indicating some differences in sex-based pathways. Finally, on linear mixed model analyses, including age, sex and stimulator type, VAS decreased with age and at each timepoint, with a trend towards increasing with sex, while PPR increased at 3 and 6 months and opioid use was decreased with HF-SCS use.

Similarly to another retrospective SCS study, we did not find sustained decrease in VAS at the 12 month endpoint [27]. This is in contrast to prior clinical trials which note consistent decrease in VAS up to twelve $[6,28]$ and twenty-four months [29]. This difference may be due to clinical trial methodology and patient population recruited for these trials. While we do not have patient satisfaction data, prior studies demonstrate high satisfaction even without long-term SCS efficacy [30].

Next, when compared to LF-SCS, patients with HF-SCS had significant improvement in PPR, but not VAS at 3 and 6 months. These findings are in agreement with some smaller studies. De Andres et al. found no difference in pain numerical rating scale score at all time points, although the study was limited to FBSS patients [28]. A crossover study also found no difference between LF-SCS and paresthesia-free SCS although this study utilized 1kHz paradigms rather than 10khz [31]. In contrast, a randomized controlled trial found significantly greater decrease in VAS for HF-SCS compared to LF-SCS persisting to 12, which was extended to 24 months with the same finding $[6,11]$. While other studies did not evaluate PPR, we used it in addition to VAS in order to distinguish from other potential pain the patient may be having at the time of visit, unrelated to the focus of SCS treatment (e.x. migraine). This difference in pain response to LF-SCS and HF-SCS may be somewhat attributed to sex, as supported by significant decreases in PPR among females implanted with HF-SCS but not LF-SCS. Furthermore, when accounting for age, time and stimulator type, there was a trend for higher VAS among females. Prior literature reported that females were more likely to have an SCS explanted than males, due to inadequate pain relief and possibly worsening depression associated with pain [21, 23].

However, few studies have reported on sex-based differences in SCS efficacy. Kumar et al., found that females had better chances of SCS trial success, and improved pain relief in the first year; however, long-term, males had a higher success rate [32]. In contrast, a case series reported a trend towards a greater percentage of females finding relief from SCS but this difference was not statistically significant [33]. Similarly, a recent retrospective study found no differences in pain relief between males and females implanted with HF-SCS [23]. Most recently, Mekhail et al. found no differences in self-reported pain by sex at 6 and 12 months following SCS implantation [24]. Furthermore, a meta-analysis including 59 studies found no differences in pain relief according to sex [34]. The sex differences found here might be attributed to HF-SCS given the hormonal effects on pain [35] and immune mediators that suggest that males and females have different underlying pathophysiology of chronic pain [19].

Lastly, while opioid use was higher among patients with LF-SCS, opioid dosage didn't change significantly among HF-SCS or LF-SCS throughout the observed period. This is in contradisctinction with results from studies that found not only a decrease in opioid use in over half of patients, but apparently also found that approximately $30 \%$ of patients stopped taking opioids all together $[15,16,27]$. Prior studies found that decrease in opioid use is traditionally greater among HF-SCS patients [6] and is estimated to be around 25 morphine mili-equivalents [27]; however this difference in opioid use decrease was not significant in a meta-analysis [36]. Furthermore, a recent study suggests that opioid use continues to significantly decrease beyond 12 months [37]. It is unclear why our patient population did not experience a decrease in opioid dosage or why HF-SCS patients were on a significantly lower dose of opioids from the beginning; however it is possible that this is in part due to LF-SCS being an older patient cohort (prior to FDA approval of HF-SCS), in particular patients who were routinely treated with higher dosages prior the opioid epidemic. 
There are several limitations to this study. First, this study was conducted at a single tertiary-care institution, and there may be site specific baseline differences among this cohort. Next, we utilized self-reported VAS as a measure of efficacy and did not have the ability to look at functional status that may be a better representation of both objective and subjective components of pain [38]. Furthermore, not all patients filled out VAS scores and PPR at every visit; however we believe these data are missing at random. Third, we extracted morphine mili-equivalent doses from our electronic health record, and it is possible that the patients may have had additional outside opioid prescriptions, both prior to and following SCS implantation.

Some strengths of this study include a real-world analysis of SCS over ten years that reflect clinical practice, stratified both by sex and type of stimulator. Despite this being a single center study, our population size is comparable to those seen in seminal SCS studies.

\section{Conclusion}

Across all patients, HF-SCS was more effective at improving PPR and was associated with less opioid use compared to LFSCS. Females with HF-SCS demonstrated significant improvement in PPR at 3 and 6 months compared to baseline, while this was not seen in LF-SCS females. In addition, among males PPR was significantly better for HF-SCS at 3 and 6 months while this was only present at 6 months for females. Finally, LF-SCS males used more opioids post-trial and at 6 months, while females used more opioids post-trial, at 3 and 6 months, potentially indicating some differences in sex-based pathways. Further studies are needed to evaluate the impact of SCS paradigm on pain control and opioid use between the two sexes.

\section{Abbreviations}

SCS

Spinal Cord Stimulation

LF-SCS

Low Frequency Spinal Cord Stimulation

PB-SCS

Parasthesia Based Spinal Cord Stimulation

HF-SCS

High Frequency Spinal Cord Stimulation

VAS

Visual Analog Scale

PPR

Perceived Pain Reduction

FBSS

Failed Back Surgery Syndrome

CRPS

Complex Regional Pain Syndrome

\section{Declarations}

Ethics approval and consent to participate:

This study was approved by the institutional review board at the University of California San Diego (IRB\#20031).

Consent for publication: 
Not Applicable

Availability of data and material:

The dataset analyzed in this study is available from the corresponding author on reasonable request.

Competing interests:

IL has been a paid consultant for Boston Scientific and received investigator initiated grants from Boston Scientific and Nevro Corporation. All other others claim no competing interests.

\section{Funding:}

IL is funded by the Brain and Behavior Research Foundation: Award 2291, funded in part by Veterans Affairs, Career Development Program - Panel I (RRD8) \#1IK2RX002920-01A1.

\section{Authors' contributions:}

RRZC helped with final data validation, methodology selection, analyzed and interpreted the patient data, prepared some of the visualizations and wrote a significant portion of the original draft. JC conceptualized the project, performed data curation and validation, and helped with the original draft and final editing. CLC performed data curation and validation, and helped with the original draft. ZR performed data curation and validation, and helped with the original draft. EN performed data curation and validation, performed preliminary analysis and edited the final draft. SY prepared and conceptualized visualizations, helped with original drafting of the paper. IL acquired funding, conceptualized the project, established methodology, supervised the project, and reviewed and edited the manuscript. All authors read and approved the final manuscript.

\section{Acknowledgements:}

Not Applicable

\section{References}

1. Dahlhamer J, Lucas J, Zelaya C, Nahin R, Mackey S, DeBar L, Kerns R, Von Korff M, Porter L, Helmick C. Prevalence of Chronic Pain and High-Impact Chronic Pain Among Adults - United States, 2016. MMWR Morb Mortal Wkly Rep. 2018;67:1001-6.

2. Turk DC, Audette J, Levy RM, Mackey SC, Stanos S. Assessment and treatment of psychosocial comorbidities in patients with neuropathic pain. Mayo Clin Proc. 2010;85:42-50.

3. Chou R, Fanciullo GJ, Fine PG, Adler JA, Ballantyne JC, Davies P, Donovan MI, Fishbain DA, Foley KM, Fudin J, et al. Clinical guidelines for the use of chronic opioid therapy in chronic noncancer pain. J Pain. 2009;10:113-30.

4. Volkow ND, McLellan AT. Opioid Abuse in Chronic Pain-Misconceptions and Mitigation Strategies. N Engl J Med. 2016;374:1253-63.

5. Dones I, Levi V. Spinal Cord Stimulation for Neuropathic Pain: Current Trends and Future Applications. Brain Sci $2018,8$.

6. Kapural L, Yu C, Doust MW, Gliner BE, Vallejo R, Sitzman BT, Amirdelfan K, Morgan DM, Brown LL, Yearwood TL, et al. Novel 10-kHz High-frequency Therapy (HF10 Therapy) Is Superior to Traditional Low-frequency Spinal Cord Stimulation for the Treatment of Chronic Back and Leg Pain: The SENZA-RCT Randomized Controlled Trial. Anesthesiology. 2015;123:851-60.

7. Melzack R, Wall PD. Pain mechanisms: a new theory. Science. 1965;150:971-9. 
8. Caylor J, Reddy R, Yin S, Cui C, Huang M, Huang C, Ramesh R, Baker DG, Simmons A, Souza D, et al: Spinal cord stimulation in chronic pain: evidence and theory for mechanisms of action. Bioelectron Med 2019, 5.

9. Kumar K, Hunter G, Demeria D. Spinal cord stimulation in treatment of chronic benign pain: challenges in treatment planning and present status, a 22-year experience. Neurosurgery. 2006;58:481-96.

10. Al-Kaisy A, Palmisani S, Smith TE, Carganillo R, Houghton R, Pang D, Burgoyne W, Lam K, Lucas J. Long-Term Improvements in Chronic Axial Low Back Pain Patients Without Previous Spinal Surgery: A Cohort Analysis of 10-kHz High-Frequency Spinal Cord Stimulation over 36 Months. Pain Med, 19:1219-1226.

11. Kapural L, Yu C, Doust MW, Gliner BE, Vallejo R, Sitzman BT, Amirdelfan K, Morgan DM, Yearwood TL, Bundschu R, et al: Comparison of 10-kHz High-Frequency and Traditional Low-Frequency Spinal Cord Stimulation for the Treatment of Chronic Back and Leg Pain: 24-Month Results From a Multicenter, Randomized, Controlled Pivotal Trial. Neurosurgery, 79:667-677.

12. Rapcan R, Mlaka J, Venglarcik M, Vinklerova V, Gajdos M, Illes R. High-frequency - Spinal Cord Stimulation. Bratisl Lek Listy. 2015;116:354-6.

13. Russo M, Verrills P, Mitchell B, Salmon J, Barnard A, Santarelli D. High Frequency Spinal Cord Stimulation at $10 \mathrm{kHz}$ for the Treatment of Chronic Pain: 6-Month Australian Clinical Experience. Pain Physician. 2016;19:267-80.

14. North JM, Hong KJ, Cho PY. Clinical Outcomes of 1 kHz Subperception Spinal Cord Stimulation in Implanted Patients With Failed Paresthesia-Based Stimulation: Results of a Prospective Randomized Controlled Trial. Neuromodulation. 2016;19:731-7.

15. Al-Kaisy A, Van Buyten JP, Smet I, Palmisani S, Pang D, Smith T. Sustained effectiveness of $10 \mathrm{kHz}$ high-frequency spinal cord stimulation for patients with chronic, low back pain: 24-month results of a prospective multicenter study. Pain Med. 2014;15:347-54.

16. Van Buyten JP, Al-Kaisy A, Smet I, Palmisani S, Smith T: High-frequency spinal cord stimulation for the treatment of chronic back pain patients: results of a prospective multicenter European clinical study. Neuromodulation 2013, 16:5965; discussion 65-56.

17. Fillingim RB, King CD, Ribeiro-Dasilva MC, Rahim-Williams B, Riley JL 3. Sex, gender, and pain: a review of recent clinical and experimental findings. J Pain. 2009;10:447-85. rd. .

18. Sorge RE, LaCroix-Fralish ML, Tuttle AH, Sotocinal SG, Austin JS, Ritchie J, Chanda ML, Graham AC, Topham L, Beggs S, et al. Spinal cord Toll-like receptor 4 mediates inflammatory and neuropathic hypersensitivity in male but not female mice. J Neurosci. 2011;31:15450-4.

19. Sorge RE, Mapplebeck JC, Rosen S, Beggs S, Taves S, Alexander JK, Martin LJ, Austin JS, Sotocinal SG, Chen D, et al. Different immune cells mediate mechanical pain hypersensitivity in male and female mice. Nat Neurosci.

2015;18:1081-3.

20. Paller CJ, Campbell CM, Edwards RR, Dobs AS. Sex-based differences in pain perception and treatment. Pain Med. 2009;10:289-99.

21. Slyer J, Scott S, Sheldon B, Hancu M, Bridger C, Pilitsis JG: Less Pain Relief, More Depression, and Female Sex Correlate With Spinal Cord Stimulation Explants. Neuromodulation 2019.

22. Van Buyten JP, Wille F, Smet I, Wensing C, Breel J, Karst E, Devos M, Poggel-Kramer K, Vesper J. Therapy-Related Explants After Spinal Cord Stimulation: Results of an International Retrospective Chart Review Study. Neuromodulation. 2017;20:642-9.

23. Bretherton B, de Ridder D, Crowther T, Black S, Whelan A, Baranidharan G. Men and Women Respond Equally Well to Spinal Cord and Dorsal Root Ganglion Stimulation. Neuromodulation.

24. Mekhail N, Costandi S, Saweris Y, Armanyous S, Chauhan G. Impact of biological sex on the outcomes of spinal cord stimulation in patients with chronic pain. Pain Pract. 
25. Calculating Total Daily Dose of Opioids for Safer Dosage

[https://www.cdc.gov/drugoverdose/pdf/calculating_total_daily_dose-a.pdf].

26. R Core Team. R: A language and environment for statistical computing. In: 4.1.1 edition. Vienna: R Foundation for Statistical Computing; 2020.

27. DiBenedetto DJ, Wawrzyniak KM, Schatman ME, Kulich RJ, Finkelman M. 10 kHz spinal cord stimulation: a retrospective analysis of real-world data from a community-based, interdisciplinary pain facility. J Pain Res. 2018;11:2929-41.

28. De Andres J, Monsalve-Dolz V, Fabregat-Cid G, Villanueva-Perez V, Harutyunyan A, Asensio-Samper JM, Sanchis-Lopez N. Prospective, Randomized Blind Effect-on-Outcome Study of Conventional vs High-Frequency Spinal Cord Stimulation in Patients with Pain and Disability Due to Failed Back Surgery Syndrome. Pain Med. 2017;18:2401-21.

29. Kumar K, Taylor RS, Jacques L, Eldabe S, Meglio M, Molet J, Thomson S, O'Callaghan J, Eisenberg E, Milbouw G, et al. The effects of spinal cord stimulation in neuropathic pain are sustained: a 24-month follow-up of the prospective randomized controlled multicenter trial of the effectiveness of spinal cord stimulation. Neurosurgery. 2008;63:762-70. discussion 770.

30. Kemler MA, de Vet HC, Barendse GA, van den Wildenberg FA, van Kleef M. Effect of spinal cord stimulation for chronic complex regional pain syndrome Type I: five-year final follow-up of patients in a randomized controlled trial. J Neurosurg. 2008;108:292-8.

31. Duse G, Reverberi C, Dario A. Effects of Multiple Waveforms on Patient Preferences and Clinical Outcomes in Patients Treated With Spinal Cord Stimulation for Leg and/or Back Pain. Neuromodulation. 2019;22:200-7.

32. Kumar K, Hunter G, Demeria D: Spinal cord stimulation in treatment of chronic benign pain: challenges in treatment planning and present status, a 22-year experience. Neurosurgery 2006, 58:481-496; discussion 481-496.

33. Fiume D, Sherkat S, Callovini GM, Parziale G, Gazzeri G. Treatment of the failed back surgery syndrome due to lumbosacral epidural fibrosis. Acta Neurochir Suppl. 1995;64:116-8.

34. Taylor RS, Desai MJ, Rigoard P, Taylor RJ. Predictors of pain relief following spinal cord stimulation in chronic back and leg pain and failed back surgery syndrome: a systematic review and meta-regression analysis. Pain Pract. 2014;14:489-505.

35. Sherman JJ, LeResche L. Does experimental pain response vary across the menstrual cycle? A methodological review. Am J Physiol Regul Integr Comp Physiol. 2006;291:R245-56.

36. Pollard EM, Lamer TJ, Moeschler SM, Gazelka HM, Hooten WM, Bendel MA, Warner NS, Murad MH. The effect of spinal cord stimulation on pain medication reduction in intractable spine and limb pain: a systematic review of randomized controlled trials and meta-analysis. J Pain Res, 12:1311-1324.

37. Feng H, Doherty P, Rotte A. Decreased Opioid Consumption and Durable Pain Relief in Patients Treated with $10 \mathrm{kHz}$ SCS: A Retrospective Analysis of Outcomes from Single-Center. J Pain Res, 14:2593-2600.

38. Gloth FM 3rd, Scheve AA, Stober CV, Chow S, Prosser J. The Functional Pain Scale: reliability, validity, and responsiveness in an elderly population. J Am Med Dir Assoc. 2001;2:110-4.

\section{Figures}




\section{Sex Responses to Pain}
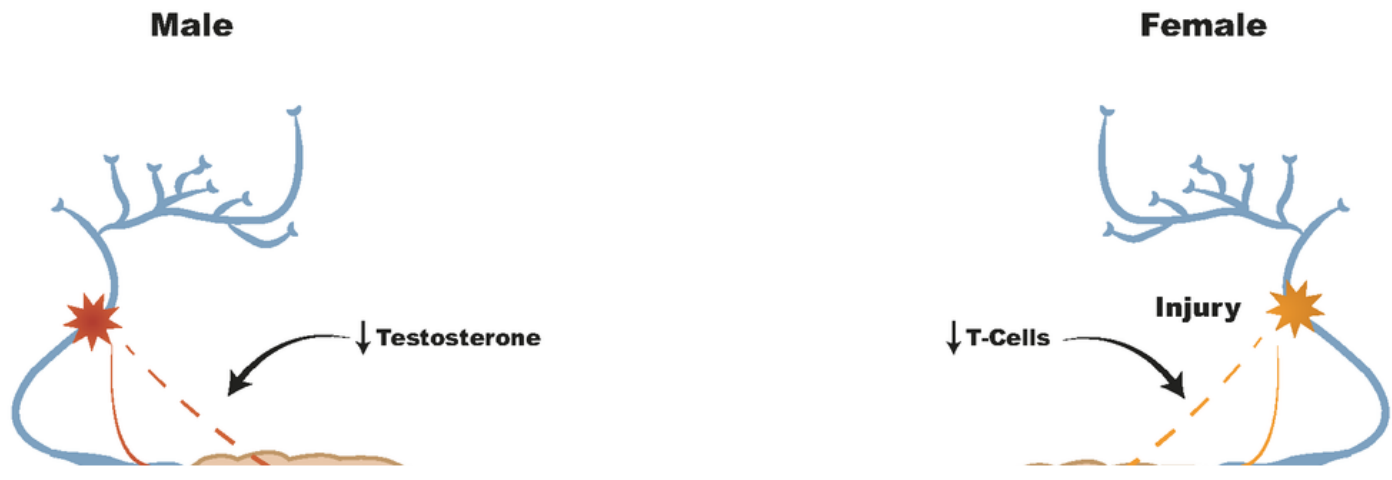

\section{Figure 1}

Sex-based differences in pain pathways. In the male model, microglia are activated to transmit pain, mediated by testosterone levels, while in the female model, the pain pathway is T-cell mediated. If a male has low testosterone levels, the pain pathway switches to the T-cell mediated pathway. Conversely, if a female takes exogenous testosterone, or if she has low T-cell levels, the pain pathway will switch to the microglia pathway.

\section{Figure 2}

Visual Analog Scale Pain Scores, Perceived Pain Reduction and Morphine Equivalent Daily Dose based on Type of Spinal Cord Stimulator 
2a. Visual analog scale pain score distribution by type of stimulator post-trial, 3 months after implant, 6 months after implant and 12 months after implant

2b. Perceived pain reduction distribution by type of stimulator post-trial, 3 months after implant, 6 months after implant and 12 months after implant

2c. Opioid use in morphine miliequivalents by type of stimulator post-trial, 3 months after implant, 6 months after implant and 12 months after implant

\section{Figure 3}

Visual Analog Scale Pain Scores, Perceived Pain Reduction and Morphine Equivalent Daily Dose based on Type of Spinal Cord Stimulator and Sex

3a. Visual analog scale pain score distribution by type of stimulator post-trial, 3 months after implant, 6 months after implant and 12 months after implant in females

3b. Visual analog scale pain score distribution by type of stimulator post-trial, 3 months after implant, 6 months after implant and 12 months after implant in males

3c. Perceived pain reduction distribution by type of stimulator post-trial, 3 months after implant, 6 months after implant and 12 months after implant in females

3d. Perceived pain reduction distribution by type of stimulator post-trial, 3 months after implant, 6 months after implant and 12 months after implant in males

3e. Opioid use in morphine miliequivalents by type of stimulator post-trial, 3 months after implant, 6 months after implant and 12 months after implant in females

3f. Opioid use in morphine miliequivalents by type of stimulator post-trial, 3 months after implant, 6 months after implant and 12 months after implant in males 